\title{
Audit in practice
}

\section{Medication compliance and dispensing among psychogeriatric patients}

\author{
C. G. Ballard, Registrar in Psychiatry, Walsgrave Hospital, Coventry CV2 2DX; \\ R. N. C. Mohan, Senior Registrar in Psychiatry, West Midlands Rotational Training \\ Scheme; S. HANDY, Registrar in Psychiatry, Walsgrave Hospital, Coventry \\ CV2 2DX; and D. DoDWELl, Senior Lecturer in Psychogeriatrics, University of \\ Birmingham
}

Compliance with medication regimes is fundamental to successful treatment and hence familiarity with factors influencing the level of patient compliance is essential. In relation to the psychogeriatric population poor compliance is a major and worrying problem largely neglected by researchers.

\section{The study}

Nineteen patients attending a psychogeriatric day hospital, and prescribed tablets, were included in a seven day compliance study. Six suffered from chronic functional mental illness, five from depression and one had chronic schizophrenia. The remaining 13 patients suffered from dementia and were living in the community, with input from a home help, a community psychiatric nurse and a social worker.

An estimation of compliance was made by counting each patient's tablets on two occasions one week apart. Patients' relatives and home carers were not told that a tablet count was to be performed or that a compliance study was to be undertaken. They were merely told on two occasions one week apart that the doctor wanted to see the tablets. No new advice was given regarding medication compliance over the study period.

The following variables were considered: the number of medications prescribed; the number of times that a particular medication was prescribed each day; whether the prescription was issued before or more recently than four months prior to the study; whether the medication taking was supervised by relatives or home carers; the use of day labelled blister packs; whether the medication was for physical or mental problems and whether the patients were suffering from functional or organic mental illness.

Enquiries were made of staff members to ask for examples of where they had implemented any specific measures or offered advice to either relatives or carers regarding medication supervision or other issues relating to medication compliance.

\section{Findings}

The 19 patients had a total of 51 prescribed medications. Only one patient took his medication in the exact quantity prescribed and the average medication percentage compliance was $72.1 \%$.

In analysing the results, a compliant patient was considered to be one taking the prescribed medication with $90 \%$ accuracy (Wandless et al, 1979). Medication compliance was poorer for medication prescribed more often than twice daily (Fisher's exact test $P<0.01$ ), and for those medications taken for less than four months $(P<0.01)$. Patients with functional mental illness had a significantly better level of compliance than those with organic mental illness $(P<0.05)$. The day labelled blister packs showed a non-significant trend to improve patient compliance.

There was little difference in compliance noted with increasing numbers of prescriptions which is contrary to research evidence in the mentally well elderly (Hurd, 1986). Neither the origins of the persons supervising the medication administration nor whether the medication was for physical or psychiatric problems affected the level of compliance.

Several medications which had been discontinued with all parties informed continued to be taken regularly. One of these was a beta blocker stopped because of peripheral vascular disease. Two patients had bottles of tablets rather inappropriately labelled "Take as directed by your doctor". Several patients had bottles containing more than 200 tablets and non-compliance was exaggerated in three patients because they failed to renew prescriptions.

No examples were given where relatives or home carers had received direct advice aimed to enhance 
medication compliance. Home care schedules had not been specifically arranged for the supervision of medication taking specifically for any patients and when this had occurred it was at the initiative of the home carer or by chance. Eight patients had their medication changed by the general practitioner without the awareness of psychiatric services.

\section{Comment}

This was a small study and it is possible that several of the non significant results were type 2 errors. This might explain why neither day labelled blister packs nor the number of prescribed medication had a significant impact on compliance. The study, however, suggests that several commonsense factors such as tailoring medication regimes to allow supervision, avoiding unnecessary changes in medication and a good communication between the GP and psychogeriatric services play a major part in enhancing compliance. Interestingly, the overall level of compliance for the psychogeriatric sample was similar to that found in a community sample of elderly people (Cartwright, 1988).

Several potentially dangerous situations were highlighted which included unclear labelling of bottles, the failure to renew prescriptions and patients continuing to take discontinued medication. This latter situation could only be prevented by physical removal of discontinued prescriptions from patients' houses.

Difficulties such as these are widespread and indicate that this is an important area for audit.

\section{Acknowledgements}

We would like to thank $\operatorname{Dr}$ A. W. T. McDowall (Medical Director, Walsgrave Hospital, Coventry) for his helpful comments in preparing the manuscript.

\section{References}

CARTWRIGHT, A. \& SMIth, C. (1988) Elderly People: Their Medicines and Their Doctors. London: Routledge.

HuRD, P. D. (1986) Compliance problems and the older patient. Drug Intelligence and Clinical Pharmacology, 20, 228-231.

Wandless, I., Mucklon, J. C., Smith, A. el al (1979) Compliance with prescribed medicines: A study of elderly patients in the community. Journal of the Royal College of General Practitioners, 29, 391-396.

A full list of references is available from $\mathrm{Dr}$ Ballard on request.

\title{
Clinical audit effects a reduction in routine prescribing of benzodiazepine hypnotics
}

\author{
Peter Childs, Registrar in Psychiatry, Edith Morgan Centre, Torbay Hospital, Torquay, \\ South Devon TQ2 7AA
}

The Edith Morgan Centre is a 60-bedded acute psychiatric in-patient unit on a DGH site, now open for three and a half years, following the closure of a nearby psychiatric hospital.

Each academic term for the last three terms there has been a session each week devoted to clinical audit, primarily involving medical staff, but on occasions other disciplines. Two such sessions involved the auditing of prescribing policies.

\section{The study}

Before the first audit meeting, a cross-sectional survey of the prescription charts for each in-patient was undertaken randomly and without the prescribers' prior knowledge. Two months later a second such survey was performed, again randomly and without the knowledge of the prescribers. The medical staff did not know that after the first survey there would be a second survey.

The name, dose, timing and duration of all benzodiazepines received by each patient were noted. Where the prescriptions were written as 'PRN', nursing administration sheets were consulted to determine how often the patients were receiving the benzodiazepines. Although all benzodiazepine prescriptions were considered, hypnotic usage was particularly being examined. Patients who were 